\title{
CRISPRi mediated Down regulation of SFPQ Gene Expression in Human induced Pluripotent Stem Cells Results in Massive Cell Death
}

Navya Lam

Henry M Gunn High School

Shinya Yamanaka

Gladstone Institutes

Samuel Perli ( $\square$ samuel.perli@gladstone.ucsf.edu )

Gladstone Institutes

\section{Research Article}

Keywords: iPSCs, CRISPRi, sgRNA, SFPQ, Repression, Paraspeckles

Posted Date: January 13th, 2021

DOI: https://doi.org/10.21203/rs.3.rs-139703/v1

License: (c) (i) This work is licensed under a Creative Commons Attribution 4.0 International License.

Read Full License 


\section{Abstract}

CRISPR-Cas9 is widely used for targeted genome editing for a wide range of organisms. In this study, we used CRISPR interference (CRISPRi) which employs a catalytically inactive version of Cas9 (known as Dead Cas 9 or dCas9) fused to KRAB a chromatin modifier. Similar to Cas9, the dCas 9 protein forms a complex along with single guide RNA (sgRNA) and binds desired DNA sequences in the presence of a protospacer adjacent motif (PAM). However, unlike Cas9, dCas9 doesn't cleave the DNA sequence but can repress gene expression when fused with KRAB. The role of paraspeckles in human cell biology is relatively unknown. In this study, we used CRISPRi to investigate the role of splicing factor proline and glutamine rich (SFPQ), also known as PSF (PTB-associated splicing factor), which is a conserved and core component of paraspeckles. We designed and constructed six different sgRNAs to target different locations of the SFPQ gene in Human induced Pluripotent Stem Cells (HiPSCs). The most effective sgRNA (sgRNA \#5) knocked down the expression of SFPQ up to $>99 \%$. We also observed that the knockdown of SFPQ exhibits severe cell-death phenotype in HiPSCs. This finding suggests that CRISPRi based SFPQ repression can modulate stem cell proliferation and maintenance. This study also suggests that SFPQ could play a vital role in proliferating cells such as cancer cells. Most importantly, these results are significant because in mice, knocking out another core component of paraspeckles results in no apparent phenotype despite the complete loss of paraspeckles. Given that traditional drug testing approaches rely on animal models, it is consequential to consider that human cell biology is more distinct than previously imagined. This study provides a new insight for role and function of SFPQ in iPSCs.

\section{Background}

Induced Pluripotent Stem Cells (iPSCs) are reprogrammed cells created from somatic cells. Our lab has pioneered the iPSC technology by inserting four unique transcription factors - Oct3/4, Sox2, KIf4 and Myc that convert somatic cells into pluripotent stem cells ${ }^{1}$. iPSCs serve as an excellent model for investigating human cell biology and hold a key solution for regenerative medicine because of their potential to replenish diseased or damaged cell types including heart, neurons, liver, pancreas and more ${ }^{1,2}$. Subsequently, several studies have been published globally on iPSCs with wide range applications from therapeutic to pharmaceutical science.

Although the molecular function and phenotypic roles of many human proteins have been discovered, we are still in early stages in terms of understanding the roles of several key factors. We can delineate the physiological relevance of these understudied proteins, by modulating their expression levels in their native context. iPSCs are patient-specific reprogrammed cells that can self-renew indefinitely as well as capable of generating all three germ-layers in vitro ${ }^{1,3}$. iPSCs therefore are an excellent model system for investigating and studying the role of various proteins of interest especially in the context of cell proliferation, differentiation and disease modelling ${ }^{4}$.

Paraspeckles have been recently discovered as mammalian specific nuclear bodies ${ }^{5}$. They are comprised of a long, non-coding RNA (nuclear enriched abundant transcript 1-NEAT1) as well as a handful of key 
proteins belonging to the RNA binding protein of Drosophila behaviour and human splicing (DBHS) family that assemble them. Paraspeckles are found in almost all of mammalian cells but their function is not yet determined. It has recently shown that they might play a significant role in biological process including cellular differentiation and stress response ${ }^{5}$.

Splicing Factor Proline and Glutamine Rich (SFPQ) is a conserved and core component of paraspeckles. It is an integral protein that helps with the formation and maintenance of paraspeckles in mammalian cells ${ }^{5}$. SFPQ is also speculated to play a vital role in other biological processes such as DNA repair, RNA splicing and transport ${ }^{6-9}$. In another study in mice, knocking out of the gene that encodes Nuclear Enriched Abundant Transcript 1 (NEAT1) RNA, another core component of paraspeckles, resultant in a complete loss of paraspeckles, but no apparent phenotypic changes were observed in mice ${ }^{5}$. Although the loss of SFPQ, has been shown to result in the loss of paraspeckles in other mammalian organisms, the phenotype of the loss of paraspeckles in human cells is unknown. In the present study, we have investigated the role of paraspeckles in human cells by CRISPR interference based knocking down of SFPQ in iPSCs.

In order to knock down SFPQ gene function in iPSCs, we have used the type II CRISPR-Cas9 system. Clustered regularly interspaced short palindromic repeats (CRISPRs) and CRISPR-associated proteins (Cas) are RNA-mediated adaptive immune system in bacteria and archaea that prevents infection from viruses $^{10,11}$. The CRISPR-Cas 9 technology is a rapid, simple, efficient, cost effective technology used in many organisms for targeted genome editing ${ }^{12-18}$. It has also been used for correcting mutation in genes for restoring gene functions ${ }^{19,20}$ such as hemoglobin $(H b A)$ gene $^{21,22}$, used for epigenetic modifications ${ }^{23,24}$, for down regulating gene function ${ }^{17,25-29}$ and many more applications.

CRISPR interference (CRISPRi) was developed by mutating two active regions of Cas9 (RuvC (D10A) and $\mathrm{HNH}(\mathrm{H} 840 \mathrm{~A})$ producing catalytically dead or dCas $9^{25}$. dCas 9 is catalytically inactive in cleaving the DNA but retains the binding ability to bind DNA in the presence of a Protospacer Adjacent Motif (PAM) and complementary small guide RNA (sgRNA). CRISPRi has been shown to mediate 1000 -fold repression in prokaryotes without any noticeable off target effects. Further optimization involves changing the location of sgRNA for improving downregulation ${ }^{25}$ as well as fusing transcriptional repressor domains including KRAB (Krüppel associated box) domain of Kox1, CS (Chromo Shadow) domain of HP1a, and WRPW domain of Hes $1^{30-32}$. In one relevant study, Qi et $\mathrm{al}^{25}$ employed dCas9 fused with KRAB to transfect into HEK293 cells that expressed GFP being chromosomally integrated. They observed a 5 -fold repression of GFP expression. Similarly, Gilbert et $\mathrm{al}^{28}$ have fused dCas 9 with dCas9-CS or dCas9-WRPW and found 2fold repression of GFP. When they have designed and constructed multiple sgRNAs, they found a combination of six sgRNAs can result in a 15-fold GFP repression. CRISPRi has also been shown to be effectively functional in human iPSCs ${ }^{33}$. It has been further used for generating genome-wide sgRNA libraries that are currently available for designing sgRNAs targeting various human genes ${ }^{34}$. In our current study, we designed and built sgRNAs for down regulating SFPQ gene expression and found that the loss of SFPQ gene expression results in a severe cell-death phenotype in iPSCs. 


\section{Materials And Methods}

\section{Strain, Plasmids and Culture}

Stellar Competent Cells (Takara, 63673) that are derived from E. coli HST08 strain were used for cloning all the constructs described in this manuscript. Chemical transformation of stellar competent cells (50 $\mu \mathrm{L}$ ) was performed by incubating cells with the ligation reaction mix on ice for $30 \mathrm{~min}$ followed by heat shock at $42^{\circ} \mathrm{C}$ for 90 seconds and recovery on ice for 5 minutes. Post recovery, heat shocked bacteria were mixed with $450 \mu \mathrm{L} \mathrm{SOC}$ media ( $2 \%$ tryptone, $0.5 \%$ yeast extract, $10 \mathrm{mM} \mathrm{NaCl}, 2.5 \mathrm{mM} \mathrm{KCl}, 10 \mathrm{mM}$ $\mathrm{MgCl}_{2}, 10 \mathrm{mM} \mathrm{MgSO}_{4}$, and $20 \mathrm{mM}$ glucose) and incubated at $37^{\circ} \mathrm{C}$ for one hour. $100 \mathrm{uL}$ of the transformed cell mixture was spread with glass beads on Luria Bertani agar containing Tryptone $(10 \mathrm{~g} / \mathrm{L})$, Yeast Extract $(5 \mathrm{~g} / \mathrm{L}), \mathrm{NaCl}(10 \mathrm{~g} / \mathrm{L})$ and $15(\mathrm{~g} / \mathrm{L})$ agar plates supplemented with Carbenicillin $(75 \mu \mathrm{g} / \mathrm{mL})$. After transformation, the plated cells were incubated at $37^{\circ} \mathrm{C}$ for 16 hours. Four successfully selected recombinant bacterial colonies were picked for each transformation into $3 \mathrm{~mL}$ LB + Carbenicillin (75 $\mu \mathrm{g} / \mathrm{mL}$ ) media and grown at $37^{\circ} \mathrm{C}$ for 16 hours. Plasmid DNA was extracted from the overnight grown cultures using Qiaprep Spin Miniprep Kit (Qiagen, 27104). Purified plasmid DNA was eluted in $50 \mu \mathrm{L}$ warm nuclease free sterile water. Cloning success was ascertained via Sanger Sequencing by Quintara Biosciences using the following sequencing primer - 5'-CAATGGGCGGGGGTCGTTGGGCGGTC-3'. The accuracy of cloning was verified by aligning the sequencing reads with expected constructs using the sequence alignment tool in the bioinformatics software - Geneious.

\section{Design and construction of plasmids}

The sgRNA expression plasmid, PB-U6-CNCB was obtained from the Conklin lab (Addgene, Plasmid \#104537). The PB-U6-CNCB vector when integrated in to the human genome via piggybac transposase constitutively expresses the sgRNAs from the mouse U6 promoter. The top six sgRNA candidates that target the SFPQ gene were chosen based on Weissman lab hCRISPRi v2 library ${ }^{34}$. The list of sgRNAs that were chosen in our study are also described in Table 1. In order to clone the sgRNAs, 23 bases forward and reverse complementary oligos for each sequence were ordered from Integrated DNA Technologies, USA (IDT) with the 5-prime overhangs 5'-TTG-3' and 5'-AAA-3' for the forward and reverse oligos respectively. To anneal, the forward and reverse complementary oligos were resuspended together at 20 $\mu \mathrm{M}$ in the TE buffer and boiled at $95^{\circ} \mathrm{C}$ for 5 minutes followed by cooling at room temperature. The annealed oligos were mixed at 6:1 molar ratio with BsmBI-v2 (New England Biolabs, R0739S) digested PB-U6-CNCB vector and ligated with 0.5 unit of T4 ligase (New England Biolabs, M0202S) at $16^{\circ} \mathrm{C}$ for 10 min and chemically transformed in to a vial of Stellar Competent Cells.

\section{iPSC culture and nucleofection}

The 1B-4 CRISPRi iPSCs were cultured on $10 \mathrm{~cm}$ laminin-coated tissue culture dishes with STEMFIT media (Ajinomoto, StemFit Basic02) supplemented with Fibroblast Growth Factor (FGF, Peprotech \# 10018C). iPSCs were passaged at $80 \%$ confluency by washing with PBS, dissociating with TrypLE Select 
Enzyme and scraping with cell scrapers. For regular maintenance, $800 \mathrm{k}$ cells are seeded for every passage on to a laminin-coated $10 \mathrm{~cm}$ dish. Nucleofection of sgRNA expressing plasmids was performed via co-transfection on 1 million resuspended cells along with a Piggybac expressing plasmid (Systems Biosciences PB210PA-1). Specifically, $7 \mu \mathrm{g}$ of Maxiprepped, endotoxin-free sgRNA expressing plasmid was added to $3 \mu \mathrm{L}$ of Piggybac expressing plasmid and mixed well along with $100 \mathrm{uL}$ of transfection reagent from the Human Stem Cell Nucleofector Kit 1 from Lonza (Lonza, VPH-5012). 1B-4 iPSCs resuspended in the nucleofection reagent containing the plasmid were nucleofected using the $\mathrm{A}-023$ protocol in Amaxa Nucleofector II and plated onto $10 \mathrm{~cm}$ laminin-coated cell culture dishes at serial dilutions for picking colonies. The successfully transfected clones were selected in Blasticidin (10 $\mu \mathrm{g} / \mathrm{mL}$ ) (ThermoFisher, \#A1113903) for 4 days. After growing to a sizable colony size, clones were picked using a P-20 pipette tip and cultured till confluency moving from a 24 well dish and on to a 6 well dish.

\section{RNA preparation and quantitative PCR}

Four days following the addition of Doxycycline $(1 \mu \mathrm{g} / \mathrm{mL})$ to the media, $1 \mathrm{~B}-4$ iPSCs containing the sgRNA expressing vectors from one confluent well of a six-well dish (about 2 million cells) were washed with PBS and collected in Qiazol (Qiagen \#217004). RNA was purified from the Qiazol collected cells using the miRNeasy mini kit (Qiagen \#217004). RNA preparations were incubated with DNase I (Qiagen \#79254) during the purification to eliminate genomic DNA contamination. The purified RNA was eluted in RNase free water into $1.5 \mathrm{~mL}$ Lobind tubes (Eppendorf \#022431021). After measuring the concentration of purified RNA using Nanodrop, total $900 \mathrm{ng}$ of RNA was used as a template for cDNA preparation using the SuperScript ${ }^{\mathrm{TM}}$ III First-Strand Synthesis SuperMix for qRT-PCR (Invitrogen, \#11752050). The cDNA was generated using randomized hexamers supplied with the kit, incubating at $50^{\circ} \mathrm{C}$ for $50 \mathrm{~min}$ and heat inactivating at $85^{\circ} \mathrm{C}$ for 5 minutes. In total $0.5 \mu \mathrm{L}$ of the cDNA prep was used as template for qPCRs using the TaqMan Universal Master Mix II (Thermo, \#4440040). A $10 \mu \mathrm{L}$ qPCR reaction consisted of $5 \mu \mathrm{L}$ 2X Taqman Universal buffer, $0.5 \mu \mathrm{L}$ cDNA prep from above, $0.5 \mu \mathrm{L}$ Taqman Probe mix (20x) and $4 \mu \mathrm{L}$ PCR-clean water. The qPCR amplification included initial denaturation at $94^{\circ} \mathrm{C}$ for $3 \mathrm{~min}$, followed by 40 cycles of denaturation at $94^{\circ} \mathrm{C}$ for 1 min, annealing of primers at $56^{\circ} \mathrm{C}$ for 30 s and extension at $72^{\circ} \mathrm{C}$ for 90s. A final extension at $72^{\circ} \mathrm{C}$ for 20 min was used. The qPCRs were set up in technical triplicates with SFPQ primer/probe mix (Hs00915444_m1) as well as the GAPDH control primer/probe mix (Hs02786624_g1) and run on Applied Biosystem's Quantstudio 3 Real-Time PCR system. The data was normalized with GAPDH control expression, analyzed and exported with Quantstudio 3 software.

\section{Results And Discussion}

SFPQ is believed to be a human tumour repression protein and play a vital role in regulating several functions in cell nucleus such as assembly of a long non-coding RNA molecule into nuclear bodies ${ }^{8}$. In addition, SFPQ has been shown to regulate key biosynthesis pathways including circadian rhythms ${ }^{35}$ and 
differentiation ability in iPSCs ${ }^{36}$. It also plays a vital role in DNA repair, splicing, transcriptional regulation and RNA transport ${ }^{37}$. In the present study, we have used CRISPRi for targeting SFPQ in human iPSCs. To this extent, we designed six different sgRNA for targeting different regions of the SFPQ gene (Fig. 1), both upstream and downstream of the Transcription Start Site (TSS) as well both, the coding and non-coding strands. The sgRNA sequences were chosen based on high ranking sgRNAs scored by the Weissman's Lab hCRISPRi v2 library ${ }^{34}$. The sgRNAs were cloned into human expression vector PB-U6-CNCB with the sgRNA being transcribed via mouse U6 promoter $^{33}$.

We introduced sgRNA expressing vectors into 1B-4 CRISPRi iPSC line through nucleofection and performed clonal selection. Since the 1B-4 cell line expresses dCas9-KRAB mediated by Doxycycline in an inducible fashion, we tested the knockdown efficiency of different sgRNAs by adding Doxycycline (1 $\mu \mathrm{g} / \mathrm{mL}$ ) to the media for 4 days (Fig. 2). We normalized the SFPQ gene expression to the endogenous GAPDH control as well as the control cells in which we did not introduce a 20-nt guide sequence. We found that sgRNA \#5 has shown highest knockdown efficiency up to $>99 \%$ and it was chosen for further analysis. We believe the highest repression achieved by sgRNA \#5 was due to its closest proximity to the transcription start site (TSS) amongst all other designed sgRNAs (Fig. 1).

Our finding underlines the important role that distance from TSS plays in sgRNA knockdown efficiency as has been noted by different studies ${ }^{34}$. When CRISPRi was used in eukaryotic HEK293 cells to repress eGFP (enhanced green fluorescent protein) ${ }^{25}$ expression, the knockdown efficiency was observed to correlates well with each sgRNA's distance from TSS, with less distance from the TSS producing the highest knockdown efficiency. We did not notice any direct effect of the orientation of sgRNA on knockdown efficiency. We also found that sgRNA \#6 which targets upstream of the TSS, surprisingly resuls in the upregulation of SFPQ gene expression. This unexpected increase in gene expression mediated by sgRNA \#6 might suggest unknown chromatin effects while targeting the upstream region of SFPQ gene. We proceeded with sgRNA \#5 and performed a growth phenotype assay of SFPQ knockdown by extending the treatment with Doxycycline for 6 days. We observed a severe cell death phenotype upon the loss of SFPQ gene function (Fig. 3). In support of our study, Hosokawa et al ${ }^{38}$ have investigated the SFPQ gene, which they knocked out in mice and found reduction in muscle mass and metabolic myopathy. However, no one has investigated the role of SFPQ in iPSCs to day. Our finding signifies an indispensable role of SFPQ/paraspeckles in the proliferation of human iPSCs.

\section{Conclusions}

Our results indicate a strong cell death phenotype associated with the knockdown of SFPQ in human iPSCs. This is a striking observation given that the loss of NEAT1 and paraspeckles in mice has no obvious phenotype. Our results warrant additional investigation into the molecular function of SFPQ as well as a potential human-specific role for paraspeckles. We also speculate that SFPQ could play an indispensable role in other proliferating cells, including cancer as well as differentiated cell types. Our work further underscores the importance of human models while developing pharmaceutical drugs. Our 
results in human iPSCs stand in stark contrast with mice studies. Given that traditional drug testing approaches rely on animal models, it is important to note that human cell biology might be more distinct than previously imagined.

\section{Declarations}

\section{Conflict of Interest}

There is no conflict of interest.

\section{Author Contributions}

N.L.L. designed and tested sgRNAs and performed phenotype assays. N.L.L., S.D.P. and SY designed experiments, analysed data, and wrote the paper.

\section{References}

1. Takahashi, K. \& Yamanaka, S. Induction of pluripotent stem cells from mouse embryonic and adult fibroblast cultures by defined factors. Cel/126, 663-676 (2006).

2. Mahla, R. S. Stem Cells Applications in Regenerative Medicine and Disease Therapeutics. Int J Cell Bio/2016, 6940283 (2016).

3. Takahashi, K. et al.Induction of pluripotent stem cells from adult human fibroblasts by defined factors. Cel/131, 861-872 (2007).

4. Rowe, R. G. \& Daley, G. Q. Induced pluripotent stem cells in disease modelling and drug discovery. Nat Rev Genet20, 377-388 (2019).

5. Nakagawa, S. \& Hirose, T. Paraspeckle nuclear bodies--useful uselessness? Cell Mol Life Sci69, 3027-3036 (2012).

6. Gozani, O., Patton, J. G. \& Reed, R. A novel set of spliceosome-associated proteins and the essential splicing factor PSF bind stably to pre-mRNA prior to catalytic step II of the splicing reaction. EMBO J13, 3356-3367 (1994).

7. Montecucco, A. \& Biamonti, G. Pre-mRNA processing factors meet the DNA damage response. Front Genet4, 102 (2013).

8. Lee, M. et al.The structure of human SFPQ reveals a coiled-coil mediated polymer essential for functional aggregation in gene regulation. Nucleic Acids Res43, 3826-3840 (2015).

9. Lim, Y. W., James, D., Huang, J. \& Lee, M. The Emerging Role of the RNA-Binding Protein SFPQ in Neuronal Function and Neurodegeneration. Int J Mol Sci21, (2020).

10. Barrangou, R. et al.CRISPR provides acquired resistance against viruses in prokaryotes. Science315, 1709-1712 (2007). 
11. Horvath, P. \& Barrangou, R. CRISPR/Cas, the immune system of bacteria and archaea. Science327, 167-170 (2010).

12. Jinek, M. et al.A programmable dual-RNA-guided DNA endonuclease in adaptive bacterial immunity. Science337, 816-821 (2012).

13. Cong, L. et al.Multiplex genome engineering using CRISPR/Cas systems. Science339, 819-823 (2013).

14. Jiang, W., Bikard, D., Cox, D., Zhang, F. \& Marraffini, L. A. RNA-guided editing of bacterial genomes using CRISPR-Cas systems. Nature Biotechnology31, 233-239 (2013).

15. DiCarlo, J. E. et al.Genome engineering in Saccharomyces cerevisiae using CRISPR-Cas systems. Nucleic Acids Res41, 4336-4343 (2013).

16. Jakočiūnas, T. et al.Multiplex metabolic pathway engineering using CRISPR/Cas9 in Saccharomyces cerevisiae. Metab Eng28, 213-222 (2015).

17. Singh, V., Braddick, D. \& Dhar, P. K. Exploring the potential of genome editing CRISPR-Cas 9 technology. Gene599, 1-18 (2017).

18. Singh, V., Gohil, N., Ramírez García, R., Braddick, D. \& Fofié, C. K. Recent Advances in CRISPR-Cas9 Genome Editing Technology for Biological and Biomedical Investigations. J Cell Biochem119, 81-94 (2018).

19. Long, C. et al.Prevention of muscular dystrophy in mice by CRISPR/Cas9-mediated editing of germline DNA. Science345, 1184-1188 (2014).

20. Nelson, C. E. et al.In vivo genome editing improves muscle function in a mouse model of Duchenne muscular dystrophy. Science351, 403-407 (2016).

21. Piel, F. B., Steinberg, M. H. \& Rees, D. C. Sickle Cell Disease. N Engl J Med376, 1561-1573 (2017).

22. Thein, S. L. Molecular basis of $\beta$ thalassemia and potential therapeutic targets. Blood Cells Mol Dis70, 54-65 (2018).

23. Hilton, I. B. et al.Epigenome editing by a CRISPR-Cas9-based acetyltransferase activates genes from promoters and enhancers. Nat Biotechno/33, 510-517 (2015).

24. Vojta, A. et al.Repurposing the CRISPR-Cas9 system for targeted DNA methylation. Nucleic Acids Res44, 5615-5628 (2016).

25. Qi, L. S. et al.Repurposing CRISPR as an RNA-guided platform for sequence-specific control of gene expression. Cel/152, 1173-1183 (2013).

26. Bikard, D. et al.Programmable repression and activation of bacterial gene expression using an engineered CRISPR-Cas system. Nucleic Acids Res41, 7429-7437 (2013).

27. Chen, B. et al.Dynamic imaging of genomic loci in living human cells by an optimized CRISPR/Cas system. Cel/155, 1479-1491 (2013).

28. Gilbert, L. A. et al.CRISPR-mediated modular RNA-guided regulation of transcription in eukaryotes. Cel/154, 442-451 (2013). 
29. Ma, H. et al.Multicolor CRISPR labeling of chromosomal loci in human cells. Proc Natl Acad Sci U S A112, 3002-3007 (2015).

30. Margolin, J. F. et al.Krüppel-associated boxes are potent transcriptional repression domains. PNAS91, 4509-4513 (1994).

31. Fisher, A. L., Ohsako, S. \& Caudy, M. The WRPW motif of the hairy-related basic helix-loop-helix repressor proteins acts as a 4-amino-acid transcription repression and protein-protein interaction domain. Mol Cell Bio/16, 2670-2677 (1996).

32. Hathaway, N. A. et al.Dynamics and memory of heterochromatin in living cells. Cel/149, 1447-1460 (2012).

33. Mandegar, M. A. et al. CRISPR Interference Efficiently Induces Specific and Reversible Gene Silencing in Human iPSCs. Cell Stem Cell 18, 541-553 (2016).

34. Horlbeck, M. A. et al.Compact and highly active next-generation libraries for CRISPR-mediated gene repression and activation. Elife5, e19760 (2016).

35. Duong, H. A., Robles, M. S., Knutti, D. \& Weitz, C. J. A molecular mechanism for circadian clock negative feedback. Science332, 1436-1439 (2011).

36. Chia, N.-Y. et al.A genome-wide RNAi screen reveals determinants of human embryonic stem cell identity. Nature468, 316-320 (2010).

37. Shav-Tal, Y. \& Zipori, D. PSF and p54(nrb)/NonO-multi-functional nuclear proteins. FEBS Lett531, 109-114 (2002).

38. Hosokawa, M. et al.Loss of RNA-Binding Protein Sfpq Causes Long-Gene Transcriptopathy in Skeletal Muscle and Severe Muscle Mass Reduction with Metabolic Myopathy. iScience13, 229-242 (2019).

\section{Tables}

Table 1. Six different 20-nt sgRNA sequences targeting human SFPQ gene at given locations.

\begin{tabular}{|lll|}
\hline Name & Sequence & Location (Chr 1, hg38) \\
sgRNA \#1 & GAGACGCTCAGGAAACGTGG & 35193086 to 35193105 \\
sgRNA \#2 & GATCGGTTCCGGAGTCGTGG & 35193021 to 35193040 \\
sgRNA \#3 & GCGGGTCCCTGAGCAACGAC & 35192703 to 35192722 \\
sgRNA \#4 & GAGAAGACGCTCAGGAAACG & 35193083 to 35193102 \\
sgRNA \#5 & GAAATGGCGGATGACACAGG & 35193125 to 35193144 \\
sgRNA \#6 & GTATAAGACGAGGACAAAGG & 35193155 to 35193174 \\
\hline
\end{tabular}

\section{Figures}




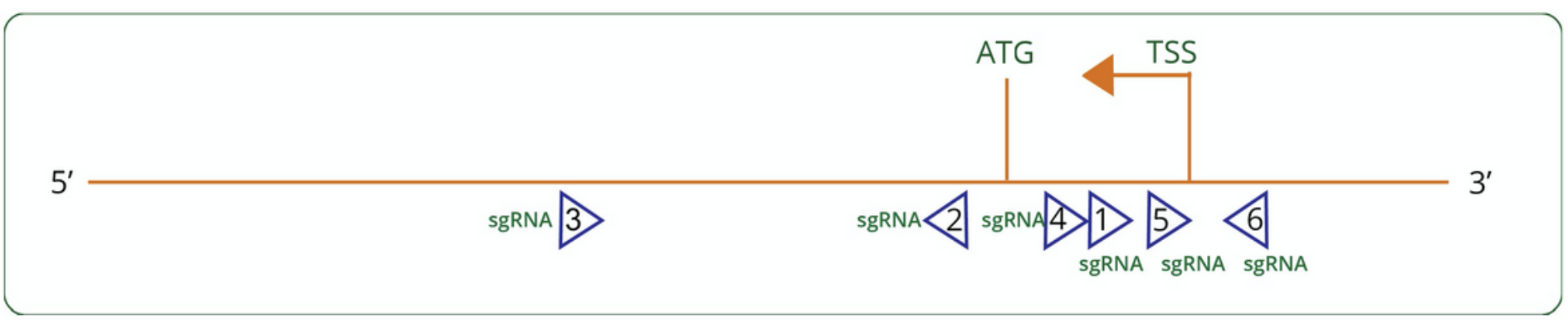

\section{Figure 1}

A schematic representation of human SFPQ gene and the targeting sgRNAs The human SFPQ gene is located on the first chromosome and is encoded in the reverse orientation. Six different sgRNAs were designed that were mapped on this gene schematic. The orientation of the sgRNAs are indicated by the pointed end of the triangle. TSS represents the Transcription Start Site while ATG represents the start codon of translation. Most of the sgRNAs chosen in this study target close to the transcription start site. 


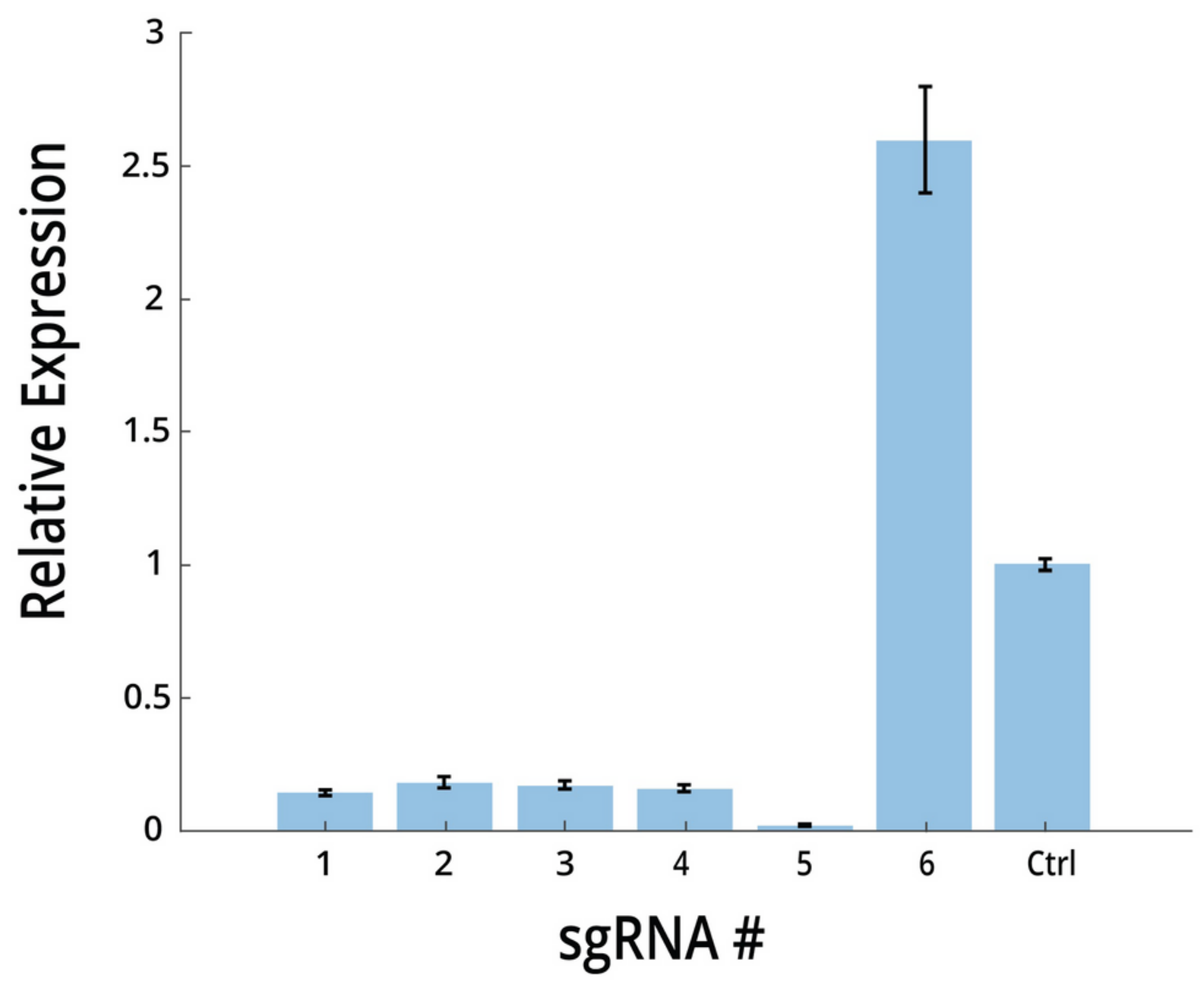

Figure 2

Knockdown of SFPQ gene expression in 1B-4 iPSCs. Six different sgRNAs were designed and introduced in to 1B-4 CRISPRi iPSCs. Ctrl represents the control, in which is an sgRNA without a 20-nt guide sequence. After Doxycycline treatment for four days, RNA was collected from the cells and cDNA was prepared. The expression level of SFPQ was probed via reverse transcription followed by quantitative PCR and reported above for each of the clones. 


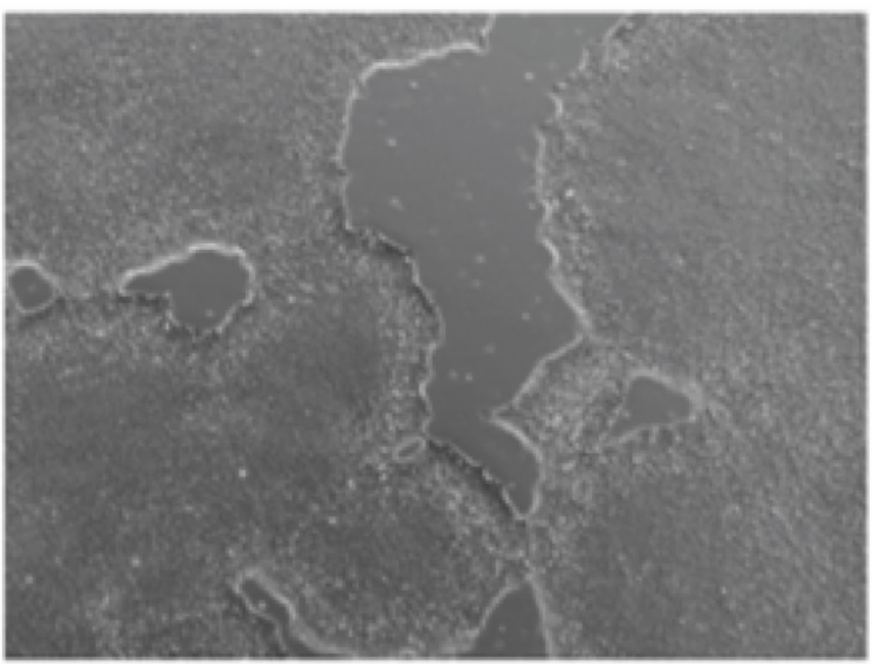

HiPSCs without SFPQ

knockdown

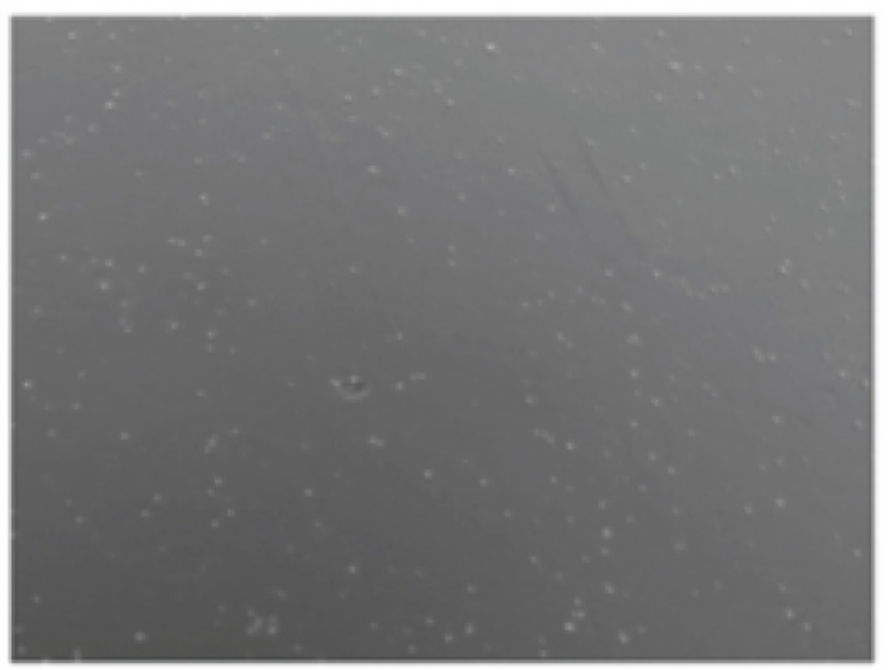

HiPSCs with SFPQ knockdown

\section{Figure 3}

SFPQ knockdown in HiPSCs results in a severe cell-death phenotype iPSC clone sgRNA \#5 that inducibly knocksdown SFPQ gene expression in the presence of Doxycycline was cultured in separate wells of a 6well dish with or without Doxycycline for 6 days. iPSCs were imaged under a light microscope and presented here. We observe a severe cell-death phenotype resulting from the knockdown of SFPQ in iPSCs. 